\title{
Percepción de estudiantes de Pedagogía sobre el desarrollo de su competencia digital a lo largo de su proceso formativo
}

\author{
Perception of students of Education on the development of their digital \\ competence throughout their learning process
}

\author{
Carolina Flores-Lueg, ${ }^{a}$ Rosabel Roig Vila ${ }^{b}$ \\ ${ }^{\text {a }}$ Departamento de Ciencias de la Educación, Universidad del Bío-Bío, Chile \\ Telf.: (56) 42 2423491. Correo electrónico: cflores@ubiobio.cl \\ ${ }^{\text {b }}$ Departamento de Didáctica General y Didácticas Específicas, Universidad de Alicante-España \\ Telf.: (34) 965903721. Correo electrónico: rosabel.roig@ua.es
}

\begin{abstract}
Se presenta un estudio cualitativo centrado en detectar la percepción de estudiantes de Pedagogía sobre la incorporación de las TIC en su proceso formativo y el significado que tiene para ellos el desarrollo de la competencia digital. Se llevaron a cabo 8 focus group donde participó un total de 54 estudiantes de la Universidad del Bío-Bío (Chile) que se encontraban realizando su práctica profesional. Para el análisis de la información se empleó un proceso de categorización mixta y el estudio de la co-ocurrencia de códigos, por medio del software Atlas.ti. v7. Los resultados evidencian que el uso de las TIC dentro de su vida estudiantil ha estado marcado por la utilización de aplicaciones tradicionales como Word, Excel o Power Point, no ocurriendo lo mismo con otro tipo de software.
\end{abstract}

Palabras clave: competencia digital, formación inicial docente, metodología cualitativa.

\begin{abstract}
A qualitative study is presented, centered in detecting the perception of pedagogy students over the incorporation of ICTs in their formative process and the meaning for them in the development of digital competence. 8 focus groups were carried out where a total amount of 54 students from Universidad del Bío-Bío (Chile) participated and who were doing their professional teaching practicum. For the analysis of the information a mixed category process was carried out and the study of co-occurrence codes by means of the software Atlas.ti.v.7. The results show that the use of ICTs in their university life has been marked by the use of traditional applications such as word, excel or power point, not happening the same with other type of software.
\end{abstract}

Key words: digital competence, teaching initial training, qualitative methodology. 


\section{INTRODUCCIÓN}

Dentro del actual escenario educativo, la formación referida al uso crítico y responsable de los medios de comunicación de masas, principalmente los más modernos, y el desarrollo de la competencia digital se constituyen en saberes clave que un egresado de cualquier ámbito o disciplina profesional debe lograr a lo largo de su proceso formativo. Estos saberes se han convertido en las herramientas que permiten a los ciudadanos participar activamente en los distintos escenarios laborales, sociales y personales que van emergiendo en la sociedad de la información, y de ahí su importancia en la formación.

La propagación de las TIC en el ámbito educativo ha dado lugar a la generación de nuevas cuestiones, nuevos desafíos y nuevas formas de enfocar los procesos formativos en todos los niveles educacionales. Dentro de este contexto, la formación inicial del profesorado ha ido adoptando cada vez mayor relevancia, pues se espera que las y los docentes sean capaces de innovar en las metodologías de enseñanza por medio de la incorporación de las herramientas digitales, las cuales pueden ser valoradas como medios que contribuyen a la generación de ambientes de aprendizaje renovados y contextualizados a la realidad que están viviendo los niños y jóvenes que asisten a la educación formal.

El docente, pues, debe apropiarse de ciertos códigos específicos, de sistemas simbólicos que van emergiendo y de formas de interactuar con la información disponible en formato digital a través de las redes de comunicación (Arras, Torres y García-Valcárcel, 2011). Por tal razón, las competencias vinculadas al tratamiento y uso de la información adoptan gran relevancia en el ámbito educativo, pero entre ellas, sin duda alguna, la competencia digital del docente se ha convertido en una de las competencias básicas del profesor del siglo XXI (Cózar y Roblizo, 2014).

$\mathrm{Al}$ analizar ciertas definiciones disponibles en la literatura sobre competencia digital se observa que en todas ellas se combinan fundamentalmente elementos ligados a la alfabetización informacional, al dominio técnico de herramientas tecnológicas y digitales. Algunas también incorporan aspectos cognitivos y comunicativos, mientras que otras consideran los aspectos éticos y legales (Gisbert, Espuny y González, 2011; Gutiérrez, 2014). Por consiguiente, se puede desprender que la competencia digital engloba un conjunto de otras competencias o subcompetencias (Adell, 2010; Cabero y Marín, 2014).

Ahora bien, al transferir lo anterior a la competencia digital del profesorado, se puede decir que esta hace referencia a la capacidad del docente para movilizar aquellas habilidades y destrezas que le permiten buscar, seleccionar críticamente, obtener y procesar información relevante haciendo uso de las TIC para transformarlas en conocimiento. Al mismo tiempo, es capaz de comunicar dicha información a través de la utilización de diferentes soportes tecnológicos y digitales, actuando con responsabilidad, respetando las normas socialmente establecidas y aprovechando estas herramientas para informarse, aprender, resolver problemas y comunicarse en distintos escenarios de interacción (Flores, 2014).

El desarrollo de la competencia digital del profesorado no es una cuestión fácil de abordar, ya que este actor debe ser capaz de desarrollar la competencia digital en cada una de sus dimensiones como cualquier ciudadano, pero, al mismo tiempo, se espera que en su praxis docente logre articular los saberes pedagógicos y disciplinarios con los conocimientos sobre el uso de las TIC desde el punto de vista didáctico, además, que valore las múltiples alternativas que estas herramientas ofrecen para generar entornos que favorezcan 
[...] aprendizajes basados principalmente en modelos participativos, apoyados en el aprendizaje colaborativo y el trabajo en grupo, con acceso a diferentes actividades y recursos educativos a través de Internet, promoviendo el aprendizaje activo y potenciando la comunicación e interacción entre personas. (Llorente, Barroso y Cabero, 2015, p. 45).

No obstante a ello, concordamos con Martínez (2009) cuando señala que estos medios "deben considerarse dentro de planes de actuación mucho más amplios donde se considere otra serie de factores que son los que al final han de justificar su presencia o no" (p. 41), porque claramente estos recursos por sí solos no mejoran los procesos de enseñanza, sino que la forma en cómo sean utilizados y el sentido que les proporcionen los docentes y discentes serán los aspectos que en alguna medida marcarán la diferencia.

El desarrollo de la competencia digital docente es un desafío que debe ser abordado en la formación inicial ya que hoy en día se requieren formas de enseñar que sean coherentes con las nuevas formas de aprender que caracterizan al siglo XXI (De Saint Pierre, 2008). En esta misma línea, diversos autores (Brun, 2011; Flores, 2014; Silva y Astudillo, 2012; Vaillant, 2013) sostienen que los planes de formación de los futuros docentes deben considerar la integración e implementación efectiva de las TIC en los procesos formativos, ya que esta instancia sería el momento propicio para que los futuros docentes egresen siendo competentes tanto en conocimientos pedagógicos y disciplinares como en conocimientos sobre las TIC aplicadas a la educación con fines didácticos.

No obstante a lo anterior, algunas investigaciones disponibles en la literatura sobre la integración de las TIC en los procesos formativos de estudiantes de Pedagogía y respecto a los niveles de aprendizaje alcanzados en cuanto a su uso didáctico, evidencian que los futuros maestros presentan insuficientes conocimientos sobre el uso de estas herramientas, que no tienen una formación específica sobre el funcionamiento de los ordenadores, que poseen destrezas a nivel básico (abrir o bajar un archivo, crear o imprimir un documento, instalar un programa o enviar un e-mail); además, se destaca el uso del correo electrónico como una herramienta de trabajo, mientras que la mensajería y las redes sociales las utilizan más en tiempos de ocio (Gutiérrez, Palacios y Torrego, 2010; Pino y Soto, 2010). En consecuencia, se podría decir que el alumnado no está tan familiarizado con las TIC como se piensa, que ignoran su potencial didáctico y las posibles formas de integración en el currículum de la enseñanza obligatoria (Gutiérrez et al., 2010; Vílchez y Pérez, 2013). Pero, por otro lado, también hay estudios que enfatizan que los futuros maestros presentan un buen nivel de conocimientos técnicos e instrumentales sobre el uso de las TIC y que dominan la mayoría de las herramientas web para la búsqueda, comunicación y colaboración (Prendes, Castañeda y Gutiérrez, 2010; Roig y Pascual, 2012), inclusive, se menciona que son los estudiantes quienes demandan una mayor formación en el uso de estas herramientas desde el inicio de su proceso formativo, tanto en la aplicación como en su integración curricular (Gallego, Gámiz y Gutiérrez, 2010; Badilla, Jiménez y Careaga, 2013).

Aunque el dominio técnico e instrumental del uso de las TIC es importante para la praxis docente, es necesario enfatizar que los programas formativos deben, además, tener en cuenta que el futuro profesor y el profesor en ejercicio necesitan adquirir conocimientos sobre cómo estas herramientas pueden favorecer el aprendizaje, cómo pueden ser aprovechadas para innovar en sus metodologías de enseñanza. En definitiva, el profesorado requiere adquirir conocimientos pedagógicos sobre el uso de TIC en el aula (Vaillant, 2013). Además, se espera que sean capaces de reflexionar sobre sus prácticas docentes con el uso de estas 
herramientas y que puedan adecuarlas a las características del contexto donde las han de utilizar, razones por las que los programas de formación docente en TIC no pueden seguir estando centrados solo en el ámbito técnico e instrumental, como ha venido sucediendo hasta hoy en día (Brun, 2011; Fainholc et al., 2013; Valverde, Garrido y Fernández, 2010).

Así pues, la formación inicial del profesorado en lo que respecta a la implementación y uso de las TIC necesita cambios profundos, pero cambios que permitan dar respuesta a las propias necesidades de los estudiantes de Pedagogía, como futuros docentes, y a las demandas que la sociedad está presentando a la educación.

En virtud de lo anterior, en este artículo se presentan los principales resultados de una investigación que estuvo centrada en detectar la percepción de estudiantes de Pedagogía sobre su nivel de competencia digital y la forma en cómo las TIC han sido incorporadas en su proceso formativo. Para ello nos propusimos los siguientes objetivos específicos:

- Definir el significado que le atribuyen los estudiantes de Pedagogía de la Universidad del Bío-Bío a los factores presentes en su proceso de formación respecto a las TIC.

- Determinar el sentido que le atribuyen los estudiantes de Pedagogía de la Universidad del Bío-Bío a los factores que intervienen en el nivel de logro de competencia digital.

\section{METODOLOGÍA}

Este estudio se llevó a cabo bajo la metodología cualitativa, considerándose como técnica para la recolección de información el focus group, con la finalidad de explorar, en un grupo de personas, "sus opiniones y actitudes sobre un tema previamente elegido por el moderador” (De Miguel, 2005 Cit. en Quintanal y García, 2012, p. 119). El uso de esta técnica buscaba explorar las opiniones y actitudes de los participantes sobre el desarrollo de la competencia digital durante el proceso de formación inicial.

Se realizaron un total de 9 grupos focales con participación de estudiantes de las carreras de Pedagogía de la Facultad de Educación y Humanidades de la Universidad del Bío-Bío (Chile), que se encontraban realizando su práctica profesional. Cada grupo correspondió a una carrera de Pedagogía distinta, a saber: Pedagogía en Educación Parvularia, Pedagogía en Educación General Básica, Pedagogía en Educación Básica con Especialidad, Pedagogía en Educación Física, Pedagogía en Educación Matemática, Pedagogía en Inglés, Pedagogía en Historia y Geografía, Pedagogía en Ciencias Naturales y Pedagogía en Castellano.

La cantidad de estudiantes que participaron en cada grupo focal se estableció teniendo en consideración lo planteado por Quintanal y García (2012) cuando señalan que "para que la comunicación sea fluida, consistente, contrastable y defendible, el número ideal de participantes oscila entre 5 y 10 miembros" (p. 121). Por tal razón, cada grupo estuvo conformado entre un mínimo de 5 y un máximo de 10 estudiantes (ver Tabla 1).

La selección de los participantes de cada grupo se llevó a cabo a partir de los siguientes criterios:

- Ser estudiante de Pedagogía de la Facultad de Educación de la UBB.

- Haber comenzado con la práctica profesional antes de ser fijada la reunión entre las investigadoras y los estudiantes.

- Tener disposición y voluntad para participar en la reunión. 
Tabla1. Detalle del número de estudiantes que han participado en los focus group

\begin{tabular}{|l|c|c|}
\hline \multicolumn{1}{|c|}{ Carrera } & $n$ & Duración \\
\hline Pedagogía en Educación Parvularia & 9 & $50^{`}$ \\
\hline Pedagogía en Educación General Básica & 5 & $50^{`}$ \\
\hline Pedagogía en Educación Básica con Especialidad & 7 & $45^{`}$ \\
\hline Pedagogía en Educación Física & 7 & $40^{`}$ \\
\hline Pedagogía en Educación Matemática & 5 & $42^{`}$ \\
\hline Pedagogía en Ciencias Naturales & 6 & $40^{`}$ \\
\hline Pedagogía en Castellano & 5 & $43^{`}$ \\
\hline Pedagogía en Inglés & 5 & $50^{`}$ \\
\hline Pedagogía en Historia & 5 & $50^{`}$ \\
\hline \multicolumn{1}{|c|}{ Total } & 54 & \\
\hline
\end{tabular}

Para reclutar a los estudiantes y desarrollar cada focus group, en primer lugar, se tomó contacto con cada Director/a de Escuela, a quien se le explicó el objetivo de la investigación y se solicitó su colaboración para contactar con los alumnos. Una vez que la investigadora tomó contacto con los estudiantes se determinó el día, hora y lugar de cada reunión.

Al inicio de cada encuentro con los estudiantes se aclararon los objetivos, se planteó el tema a abordar ("TIC aplicadas a su proceso formativo") y se les solicitó autorización para grabar en formato de video el desarrollo de la reunión. Durante el momento de diálogo o discusión se formularon preguntas semiestructuradas con formato de respuesta abierta. Estas preguntas sirvieron para orientar la conversación con los estudiantes y obtener la información requerida para la investigación.

El protocolo elaborado para generar el diálogo colectivo con cada grupo estuvo constituido por las siguientes preguntas:

- ¿Qué representan para ustedes las TIC en su vida personal?

- ¿Qué representan para ustedes las TIC en el desarrollo de su profesión?

- ¿Cómo se sienten de preparados para integrar las TIC en sus prácticas docentes en este momento y en el futuro?

- ¿Qué fortalezas y debilidades encuentran dentro su proceso formativo respecto a las TIC?

Cabe subrayar que las preguntas fueron flexibles, pues, conforme a las respuestas que los participantes iban proporcionado, se plantearon otras que a juicio de las investigadoras fueron relevantes para profundizar en ciertos aspectos de interés y para dar repuesta al objetivo planteado.

El diálogo generado a partir de la interacción entre el grupo de estudiantes y las investigadoras fue grabado en formato video y luego transcrito de forma literal a formato texto, sin modificar ni alterar el discurso oral emitido por los estudiantes participantes. 
La validez de la información obtenida fue controlada por medio de la triangulación de datos. Principalmente se consideró el contraste entre el discurso de cada uno de los estudiantes (intra-grupo), enfatizando en las divergencias y convergencias entre ellos, pues los participantes respondieron a las mismas preguntas. Posteriormente se llevó a cabo el contraste entre los grupos (inter-grupos), de este modo se pudo evidenciar la credibilidad de la información proporcionada por los participantes (Bisquerra, 2014).

Desde el punto de vista ético, se respetó plenamente la autonomía de cada uno de los participantes en los focus group, pues se les informó claramente de los fines que se pretendían con la investigación y se les invitó a participar en forma voluntaria. Además, se solicitó la autorización correspondiente para llevar a cabo el estudio en el contexto seleccionado al Decano de la Facultad de Educación y Humanidades de la Universidad del Bío-Bío y a cada uno de los Directores y Directoras de las distintas Escuelas de Pedagogía. Por otro lado, a los estudiantes que participaron en el estudio se les garantizó la protección de la identidad y se les pidió autorización para realizar la grabación en formato video de cada encuentro, asegurándoles que dicho material solo sería utilizado en forma interna a la investigación y que en ningún caso sería utilizado con otra finalidad que la señalada o difundidos en algún medio o red de comunicación.

Para llevar a cabo los análisis de los datos se establecieron las siguientes categorías semánticas:

- $\quad$ Niveles de competencias TIC: valoración de los estudiantes de Pedagogía respecto al nivel de competencias que sienten poseer respecto al manejo de las herramientas digitales, tanto en su vida personal y académica, como en cuanto a su utilización dentro del aula como recurso didáctico. La operacionalización de esta categoría se presenta en la Tabla 2.

Tabla 2. Operacionalización categoría Nivel de competencia en TIC

\begin{tabular}{|l|l|l|}
\hline Categoría & Subcategoría & Código \\
\hline \multirow{4}{*}{$\begin{array}{l}\text { Nivel de competencia } \\
\text { digital }\end{array}$} & $\begin{array}{l}\text { NCG: Conocimiento sobre } \\
\text { el uso general de TIC }\end{array}$ & $\begin{array}{l}\text { NCG1. Uso de editores de Texto } \\
\text { NCG2. Planillas de cálculo } \\
\text { NCG3. Recursos multimedia } \\
\text { NCG4. Redes sociales }\end{array}$ \\
\cline { 2 - 3 } & NCD: Conocimiento & $\begin{array}{l}\text { NCD1. Software y aplicaciones educativas } \\
\text { NCD2. Dominio de la pizarra digital } \\
\text { NCD3. Uso de TIC como recurso didáctico } \\
\text { NCD4. Creación de materiales digitales } \\
\text { NCD5. Uso de plataformas virtuales }\end{array}$ \\
\hline
\end{tabular}

NCG: Nivel de competencia digital general. NCD: Nivel de competencia digital

- Factores intervinientes en los niveles de competencias TIC: consideración que poseen los estudiantes de Pedagogía sobre los factores presentes en su proceso formativo y que, en alguna medida, han intervenido en el nivel de aprendizaje que poseen sobre el uso de las TIC. La operacionalización de esta categoría se presenta en la Tabla 3. 
Tabla 3. Operacionalización categoría Factores intervinientes en competencia TIC

\begin{tabular}{|l|l|l|}
\hline Categoría & Subcategoría & Código \\
\hline \multirow{2}{*}{$\begin{array}{l}\text { Factores intervinientes } \\
\text { en los niveles de } \\
\text { competencia digital }\end{array}$} & \multirow{2}{*}{$\begin{array}{l}\text { FC: Factores } \\
\text { contextuales }\end{array}$} & FC1. Implementación de TIC en la Facultad \\
\cline { 3 - 3 } & & FC2. Accesibilidad a los recursos disponibles \\
\cline { 3 - 3 } & & FC4. Aspecticas docentes con TIC \\
\hline
\end{tabular}

A partir de estas categorías de análisis aplicadas al discurso de los focus group se derivó una serie de resultados, de los cuales se exponen los más destacables en el siguiente apartado.

\section{RESULTADOS}

\subsection{NIVEL DE COMPETENCIA DIGITAL}

Para poder aproximarse al significado que los estudiantes les atribuyen a las TIC implementadas en su proceso de formación inicial y al sentido que para ellos tienen aquellos factores que intervienen en su nivel de competencia digital, se consideró necesario, en primera instancia, detectar la percepción de estos actores sobre el uso de las TIC en su vida personal y estudiantil. Asimismo, teniendo en cuenta que estos conocimientos son de carácter instrumental y se constituyen en la base para incorporar estas herramientas en su práctica pedagógica, se planteó dentro de esta categoría complementar dicha información con el nivel de conocimiento que ellos autoperciben respecto a su uso didáctico. Por tal razón, en términos operativos se estimó necesario establecer dos subcategorías: conocimientos sobre el uso general de TIC y conocimiento didáctico sobre el uso de TIC (ver Figura 1).

Figura 1. Red de códigos categoría Nivel de Competencias en TIC

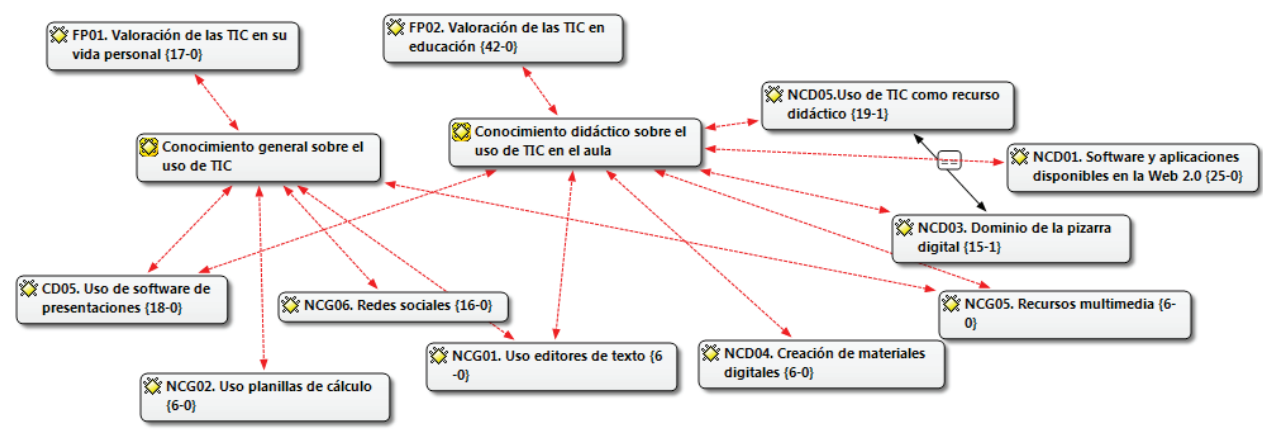

Fuente: vista de red software Atlas.ti v.7 


\subsubsection{Conocimientos sobre el uso general de TIC}

En lo que respecta a cómo el alumnado percibe su nivel de conocimientos sobre el uso general de las TIC, tanto en su vida personal como académica, se observa que tienen seguridad para manejar un procesador de textos, específicamente Word, pero destacan que el dominio de todas las herramientas que este software ofrece solo lo han ido adquiriendo con mayor profundidad en la etapa final de su proceso formativo, dadas las exigencias que les han ido presentado en su trabajo de Actividad de Titulación. Situación similar ocurre con el Power Point, pues manifiestan que los profesores les exigen realizar exposiciones haciendo uso de este programa sin enseñarles a hacer uso de todas las funciones y herramientas que posee, razón que les ha llevado a tener que aprender en forma autónoma.

Dentro de las otras herramientas que señalan saber manejar se encuentra el software de cálculo, particularmente Excel, al que accedieron en primer año de la carrera en una asignatura específica y durante el resto del periodo formativo solo algunos profesores de la carrera de Pedagogía en Ed. Matemática les solicitaban hacer análisis con este software, mientras que en las otras carreras los estudiantes señalan un uso prácticamente inexistente, por lo que no han logrado un verdadero aprendizaje sobre cómo utilizar este software. Situación similar les ocurrió con el uso de aplicaciones disponibles en la Web 2.0, particularmente con el diseño y mantenimiento de un blog (ver Tabla 4).

Tabla 4. Discurso de los estudiantes sobre su nivel de conocimientos uso general de TIC

\begin{tabular}{|l|}
\hline \multicolumn{1}{|c|}{ Extractos textuales } \\
\hline "[...] yo solamente utilizo en un computador lo que es Word, un poquito de Excel, Power Point y \\
nada más" (PC4) \\
"[...] una se ve obligada a hacer presentaciones en la Universidad, una está obligada a aprender y \\
a preguntar" (PC2). \\
"[...] el hacer un Power, pero no es que nos enseñaran cómo hacer una presentación, de Word solo \\
nos enseñaron las herramientas básicas (PC5)" \\
"Cuando llegué a la universidad ya sabía usar el Word, hacer presentaciones en Power, pero en la \\
misma línea, lo que uno aprendió mirando" (PEP7). \\
"[...] el caso de Word, que se le pueden dar muchas utilidades, uno pudiera pensar que algo tan \\
básico, pero cuando llegué acá ni siquiera podía ocupar bien ese sistema y acá se nos enseñó a \\
utilizar eso, el Power Point y muchas otras cosas también" (PEBE3).
\end{tabular}

Nota: PC: Pedagogía en Castellano y Comunicación; PEP: Pedagogía en Educación Parvularia; PEBE: Pedagogía en Educación Básica con Especialidad. Cada número que acompaña a la sigla representa al estudiante al cual se le asignó dicho número.

Por otra parte, en su vida personal destacan que las TIC son muy importantes, que para ellos el uso de estas herramientas ha pasado de ser un simple entretenimiento a una necesidad, por cuanto consideran que la sociedad en la que estamos viviendo requiere del dominio de estos recursos para poder estar comunicados y acceder a la información, tanto en su vida universitaria como en su vida personal. Es en este aspecto donde cobran pleno sentido para ellos las redes sociales, fundamentalmente Facebook, pero no solamente 
para comunicarse con sus amigos y familia, sino que esta herramienta ha adoptado una orientación comunicativo-académica, ya que gran parte de las carreras están haciendo uso de esta red social para transmitir información y/o noticias vinculadas al ámbito académico, lo que implica que si un estudiante no está conectado puede quedar al margen de los acontecimientos que ocurren en su Escuela o de la comunicación que se trasmite. Frente a ello, algunos estudiantes manifiestan que hacer uso de Facebook hoy en día se ha transformado en una necesidad (ver Tabla 5).

Tabla 5. Discurso de los estudiantes sobre la valoración que poseen respecto a las TIC y las redes sociales

\begin{tabular}{|l|}
\hline Extractos textuales \\
\hline "Principalmente la utilizamos para mantenernos conectados, sobre todo con los compañeros de la \\
Universidad para ver los correos por si llega información sobre alguna actividad, más que nada \\
eso, igual la ocupo harto, igual estoy conectada bastante tiempo" (PC2). \\
"Yo he usado las redes sociales este año solo para conversar cosas de trabajo, de práctica, hay muy \\
poco tiempo para otras cosas, pero antes uno hacía de todo por Internet, conocer gente, con los \\
amigos antiguos que no había visto" (PEP2). \\
"[...] si no tenemos Facebook o no tenemos WhatsApp y no estás relacionado es como estar \\
aislado" (PEF1). \\
"[...] yo no reviso mi Facebook en todo el día y llego a la Universidad y me dicen que tengo \\
certamen, presentación, cualquier cosa y que lo pusieron en Facebook... estoy obligado a revisar... \\
pasó de ser solo una entretención, como dijo mi compañero a una obligación para mí" (PEF2).
\end{tabular}

Nota: PC: Pedagogía en Castellano y Comunicación; PEP: Pedagogía en Educación Parvularia; PEBE: Pedagogía en Educación Básica con Especialidad; PM: Pedagogía en Educación Matemática; PEF: Estudiantes de la carrera de Pedagogía en Educación Física. Cada número que acompaña a la sigla representa al estudiante al cual se le asignó dicho número.

Los resultados inducen a sostener que, en general, para los alumnos el uso de las TIC dentro de su vida estudiantil ha estado dominada por el uso de aplicaciones tradicionales como el Word, Excel o Power Point, pues han sido las herramientas que han tenido que aprender para hacer frente a las exigencias demandadas por los docentes, no ocurriendo lo mismo con otro tipo de software, ya que solo aprendieron a utilizarlos al inicio de su periodo formativo y no volvieron a hacer uso de ellos, razón que les lleva a sentir que su aprendizaje fue poco significativo.

Finalmente, se puede apreciar una valoración positiva de las y los estudiantes frente a la presencia y uso de las TIC en su vida personal y académica. Asimismo, tienen una valoración muy positiva de la red social Facebook, porque no solamente les permite estar comunicados con amigos y/o familiares, sino que les ayuda a mantenerse informados de noticias y mensajes específicos de su carrera.

\subsubsection{Conocimientos didácticos sobre el uso de TIC}

La mayoría de los estudiantes considera que no se sienten lo suficientemente preparados para usar las herramientas tecnológicas y digitales como recurso didáctico, 
porque manifiestan que sus conocimientos son básicos y que estos se enfocan en el uso de software tradicional como Word, Power Point y Excel (ver Tabla 6).

Tabla 6. Discurso de los estudiantes sobre su nivel de conocimientos respecto al uso didáctico de las TIC

\begin{tabular}{|l|}
\hline \multicolumn{1}{|c|}{ Extractos textuales } \\
\hline "Es muy básica la capacitación que podemos tener para implementarlas en el aula, y que sea \\
entretenido y agradable para los jóvenes" (PEM1). \\
"[...] como en realidad no estamos bien capacitados no las usamos adecuadamente" (PC1). \\
"Yo creo que estoy preparado, pero hasta cierto nivel, pero creo que como profesor, educador, \\
deberíamos tener un nivel superior en el uso de las TIC, un nivel bien avanzado, porque se pueden \\
hacer cosas tan mágicas con la tecnología y a veces uno las tiene a su disposición, pero no saber \\
cómo usarlas, es casi como una persona que tiene un texto en sus manos y no sabe leer, entonces \\
no puede interpretarlo. Yo siento que si puedo hacer cosas con TIC, podría lograr muchas cosas, \\
pero no quiere decir que sepa mucho o bastante de lo que se necesita para hacer uso de ella" \\
(PEGB1). \\
"Siento que tengo conocimientos sobre lo esencial para hacer una clase interesante para ellos, \\
como un Power Point, pero como que cuesta saber lo que es interesante para los alumnos" (PI2). \\
"[...] yo en lo personal ni siquiera sé instalar el data, entonces en el colegio yo aprieto un botón \\
y se enciende, pero si yo lo tuviera que instalar y conectarlo no sabría" (PH3). \\
"yo puedo decir que me siento en un nivel básico, pero para los demás es más alto que para mí. \\
Si uno lo ve en la realidad uno ocupa Word, Excel, pero hay otros programas que van más allá y \\
uno aquí en la universidad ni siquiera los ha visto" (PEBE7). \\
\hline
\end{tabular}

Nota: PC: Pedagogía en Castellano y Comunicación; PEP: Pedagogía en Educación Parvularia; PEBE: Pedagogía en Educación Básica con Especialidad; PM: Pedagogía en Educación Matemática; PI: Pedagogía en Inglés; PH: Pedagogía en Historia y Geografía; PEG: Pedagogía en Educación General Básica. Cada número que acompaña a la sigla representa al estudiante al cual se le asignó dicho número.

En lo que respecta al conocimiento sobre software y aplicaciones educativas, solo algunos estudiantes, por iniciativa propia, han descubierto algunas más recientes, como Prezi, o han accedido a entornos de creación de materiales multimedia como el JClic, pero se observa que no es una práctica generalizada. Por otra parte, algunos destacan que en una asignatura tuvieron que diseñar un blog y una web, pero solo fue una actividad específica que no perduró en el tiempo, por lo que no se preocuparon por el mantenimiento o su actualización, e inclusive con el paso del tiempo se les olvidó cómo diseñarlos. Solo se destaca la carrera de Pedagogía en Educación Matemática, pues los estudiantes señalan que conocen algunas aplicaciones específicas para su disciplina porque han sido utilizadas por algunos docentes frecuentemente, pero señalan que no tienen la preparación para hacer uso de las funciones que estos recursos tienen, por lo que prefieren usar los programas tradicionales, aunque sean más limitados.

Una de las cosas que se observa de forma recurrente es la opinión generalizada sobre la incapacidad para usar una pizarra digital interactiva, pues declaran que no tienen conocimientos al respecto. Esta situación la justifican señalando que durante su proceso 
formativo no han tenido oportunidad de practicar con este recurso. Esta situación induce a sostener que esta herramienta ha sido escasamente valorada tanto por los docentes como por los propios estudiantes, pues solo ahora que se encuentran en la etapa de su práctica y que se han visto con la posibilidad de usarla en los centros educativos, les ha surgido la preocupación de no saber utilizarla.

En cuanto al uso de TIC como recurso didáctico en sus clases, gran parte de ellos destaca el uso de videos extraídos de YouTube como recurso para favorecer la motivación, sobre todo en los niveles de Educación Parvularia y Primer Ciclo Básico. Por otra parte, aluden de manera bastante reiterativa a la utilización de presentaciones en Power Point para explicar contenidos que estén abordando en sus clases (ver Tabla 7).

Tabla 7. Evidencia sobre alternativas didácticas del uso de TIC conocidas por los estudiantes

\begin{tabular}{|c|c|}
\hline Subcategorías & Extractos textuales \\
\hline $\begin{array}{l}\text { Conocimiento } \\
\text { sobre software y } \\
\text { aplicaciones } \\
\text { educativas }\end{array}$ & $\begin{array}{l}\text { "[...] buscaba un programa para crear un juego para los niños porque el } \\
\text { Power Point es bueno, pero igual les aburre un poco, encontré el JClip que } \\
\text { es un programa educativo y que muchos no saben que existe, entonces creé } \\
\text { una materia para pasar a través del juego y eso me sirvió mucho para mis } \\
\text { clases y a los niños les gustó" (PEBE3). } \\
\text { "Hay una gran cantidad de material que podemos usar, muchísima cantidad, } \\
\text { uno se sorprende de repente porque hay cosas que no se imagina que se } \\
\text { pueden utilizar en el aula... por eso yo mismo trato de buscar siempre" } \\
\text { (PEGB3). } \\
\text { "Hicimos un blog y una página Web grupal, pero a mí ya se me olvidó hacer } \\
\text { la página, pero el blog es más fácil (...), pero yo creo que necesitamos la } \\
\text { otra parte de cómo crear un programa para enseñar mejor" (PEBE5). }\end{array}$ \\
\hline $\begin{array}{l}\text { Dominio de la } \\
\text { Pizarra Digital } \\
\text { Interactiva }\end{array}$ & $\begin{array}{l}\text { "Yo por lo menos nunca he visto una pizarra digital, no la enseñaron a } \\
\text { utilizar ni nada. Sé que la Universidad cuenta con una, pero nadie te enseña } \\
\text { a trabajar con ella" (PC2). } \\
\text { "[...] aquí en la Universidad hay pizarras digitales, pero me acuerdo que a } \\
\text { un compañero le tocó disertar sobre eso y listo, solo él aprendió algo y lo } \\
\text { bueno hubiese sido que todos aprendiéramos algo porque en las clases nos } \\
\text { puede tocar usarla" (PC2). } \\
\text { "Yo también creo que es vergonzoso llegar a un colegio sin saber usar una } \\
\text { pizarra digital, porque puede ser que también lleguen practicantes de otras } \\
\text { universidades que sí saben usarlas y nos van a empezar a comparar y van a } \\
\text { cuestionar qué pasa, eso que son de la Universidad del Bío-Bío" (PEBE6). }\end{array}$ \\
\hline $\begin{array}{l}\text { Uso de TIC como } \\
\text { recurso didáctico } \\
\text { en clases }\end{array}$ & $\begin{array}{l}\text { "[...] por ejemplo, si uno trabaja unidades uno recurre por lo general a } \\
\text { YouTube a buscar videos didácticos, educativos para echar mano un poco } \\
\text { [...] de eso para que sea más entretenido para los niños" (PEP5). } \\
\text { "utilizo varias herramientas y también en el aula las diapositivas, las } \\
\text { presentaciones, el proyector, los videos, que a mí me encanta trabajar con } \\
\text { videos, siempre tratamos de aplicar harto lo que son las TIC en el aula, } \\
\text { cuando hacemos presentaciones aquí mismo en la Universidad nos } \\
\text { apoyamos bastante por estas herramientas" (PEGB2). }\end{array}$ \\
\hline
\end{tabular}


Creación de materiales digitales

"Yo he partido con un video, por ejemplo, y todos pegados a los videos, después de eso unas diapositivas interactivas con hipervínculos donde les ponía y ellos aplaudían, los veía felices, entonces a ellos eso les atrae, que no hay que olvidar que son niños y que eso es parte del juego" (PEGB1). "Yo también he hecho presentaciones en el programa Prezi, que igual es como más entretenido para los niños" (PEGB4).

"[...] nunca nos han hecho trabajar con Internet y crear cosas" (PEM3).

"[...], entonces yo he aprovechado de sacar recursos de Internet como gifs animados y los incorporo en las presentaciones y los niños súper fascinados" (PEP7).

Nota: PC: Pedagogía en Castellano y Comunicación; PEP: Pedagogía en Educación Parvularia; PEBE: Pedagogía en Educación Básica con Especialidad; PM: Pedagogía en Educación Matemática; PH: Pedagogía en Historia y Geografía; PEGB: Pedagogía en Educación General Básica; PCN: Pedagogía en Ciencias Naturales; PEF: Pedagogía en Educación Física. Cada número que acompaña a la sigla representa al estudiante al cual se le asignó dicho número.

\subsection{FACTORES INTERVINIENTES EN LOS NIVELES DE COMPETENCIAS TIC}

Para llevar a cabo el análisis de esta categoría, en términos operativos, se han establecido las siguientes subcategorías: Factores personales, Factores contextuales (ver Figura 2).

Figura 2. Red de códigos categoría factores intervinientes en el nivel de competencias TIC

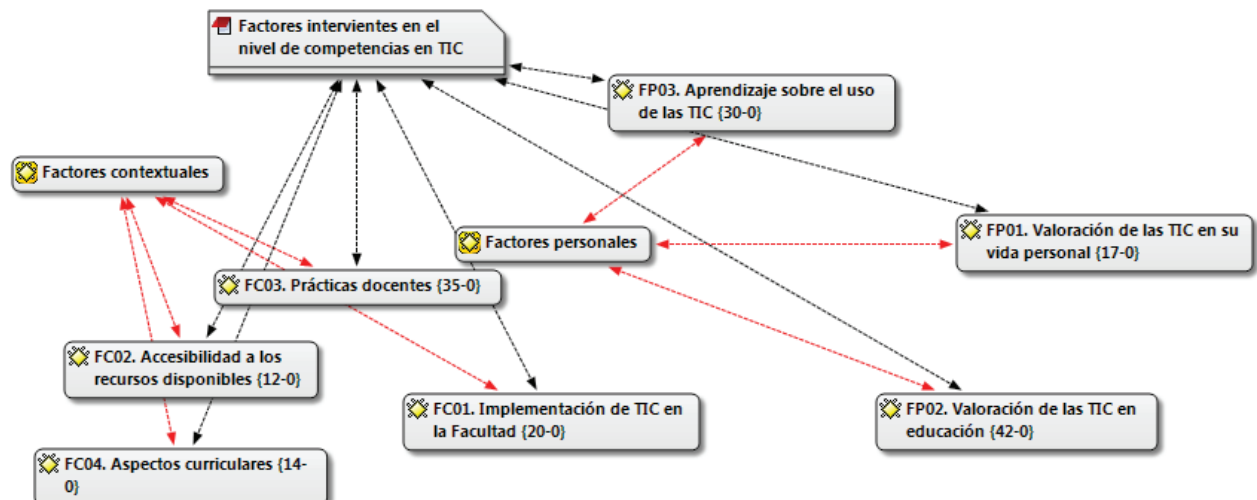

Fuente: vista de red software Atlas.ti v.7

\subsubsection{Factores personales}

Se observa una valoración muy positiva de los estudiantes respecto a las TIC, tanto en su vida personal como aplicadas a la educación. Estas herramientas han adoptado gran importancia en su vida por cuanto se han trasformado en recursos que les permiten estar comunicados y se constituyen en un medio para acceder a la información que requieren 
para cumplir con las demandas académicas. Ahora bien, se aprecia una asociación directa de los estudiantes entre el concepto TIC y el uso exclusivo de ordenadores, el uso de redes sociales, acceso a Internet y la utilización de software de Microsoft Office. Solo casos muy puntuales de estudiantes hacen mención, por ejemplo, al celular como herramienta tecnológica o señalan que hablar de TIC es ir más allá que simplemente usar el Word, Power Point o Internet.

En cuanto a las TIC aplicadas a la educación, los estudiantes consideran que estas herramientas son un aporte a la labor docente porque les permiten optimizar los tiempos y les sirven como un complemento para sus clases, fundamentalmente para motivar a los estudiantes y favorecer el aprendizaje. Se destaca el hecho de que para ellos estas herramientas son solo un medio y no un fin en sí mismas, pues sostienen que están al servicio de la labor docente $y$, por ello, consideran necesario tener competencias para otorgarles un uso educativo.

Por otra parte, llama la atención que a pesar de que el alumnado no se siente preparado para integrar didácticamente estas herramientas en el aula, como se observó anteriormente, sostienen que su implementación en la práctica docente implicaría asumir un cambio paradigmático respecto a la forma de enfrentar el proceso de enseñanza y aprendizaje. Argumentan que la sociedad en la que están viviendo ellos y los niños les exige hacer uso de las tecnologías, y esta demanda conlleva a la generación de cambios más profundos en las prácticas docentes, los que van más allá de proyectar diapositivas o actualizar los aparatos tecnológicos.

Finalmente, en esta categoría se subraya el hecho de que los estudiantes han aprendido a hacer uso de las TIC en forma autodidacta, donde también han tenido un rol importante sus pares, pero no así la formación que han recibido en la Universidad. Cabe reiterar que este aprendizaje autónomo, como se ha señalado, ha estado centrado en el uso de procesadores de texto, en software de presentaciones y algunas aplicaciones específicas para su disciplina. Todo el conocimiento sobre el uso de programas como Word, Excel, Power Point y la diversidad de herramientas que estos programas poseen lo han logrado gracias a que los docentes les han exigido su uso durante todo su proceso formativo, pero subrayan que estos no se han dedicado a enseñarles cómo hacerlo, razón que los ha llevado a la necesidad de buscar alternativas por su propia cuenta para aprender a emplear las distintas herramientas que estos software poseen (ver Tabla 8).

Tabla 8. Extractos textuales subcategorías (factores personales)

\begin{tabular}{|l|l|}
\hline Sub-categorías & \multicolumn{1}{c|}{ Extractos textuales } \\
\hline \multirow{3}{*}{$\begin{array}{l}\text { "Todo, comunicación, las uso para trabajar mucho. Mi celular sí es mi } \\
\text { compañero de toda la vida, pero en el computador no me gusta estar } \\
\text { metida en el chat, solamente lo uso para trabajar, hacer guías," (PEM). } \\
\text { en su vida personal }\end{array}$} & $\begin{array}{l}\text { "[...] en lo personal lo que más utilizo yo es para conectarme a las } \\
\text { redes sociales y ser beneficiario. Principalmente para eso, como } \\
\text { medio de comunicación, es como el fin de las redes sociales. También } \\
\text { se utiliza mucho para realizar trabajos, hacer tareas si lo llevamos a } \\
\text { otro ámbito, pero para satisfacer mi necesidad es más social, para } \\
\text { comunicarme, para participar en las redes sociales" (PEF6). }\end{array}$ \\
\hline
\end{tabular}


Valoración de las TIC aplicadas a la educación

\begin{abstract}
"[...] de que son buenas, son buenas! y obviamente hacen que, como que alivianan el peso de la pizarra, la tediosidad de la clase, lo monótono. Eso lo aliviana y a los chicos se les hace un poquito más fácil en algunos casos" (PEM2).

"Son importantes porque nos ayudan como herramienta para mantener la atención de los estudiantes, algo que cuesta mucho en este tiempo porque los jóvenes son muy inquietos y, por ejemplo, si uno les muestra alguna imagen graficando los contenidos, ellos lo aprenden más fácilmente a que uno les esté dictando, como lo hacían antes cuando nosotros mismos estábamos en el colegio. Entonces es una herramienta para que ellos puedan aprender mejor" (PC2).

"[...] facilita mucho el trabajo, se podría decir, porque, por ejemplo, yo recuerdo antes los profesores tomaban el libro de clases, sacaban las notas y con calculadora en mano tenían que sacar los promedios, ahora con programas como el Excel, por ejemplo, con una fórmula y listo, promedio sacado!... las redes sociales, Word, Power Point y todo eso sirven mucho como apoyo para el lado laboral, como docente" (PC6).

"Yo lo veo por la cercanía con la que uno llega a los estudiantes, ellos son usuarios de videos, música" (PC5).
\end{abstract}

Nota: PC: Pedagogía en Castellano y Comunicación; PM: Pedagogía en Educación Matemática; PEF: Pedagogía en Educación Física. Cada número que acompaña a la sigla representa al estudiante al cual se le asignó dicho número.

\subsubsection{Factores contextuales}

Uno de los aspectos que los estudiantes valoran ampliamente es la implementación de herramientas tecnológicas que posee la Facultad, fundamentalmente la dotación de ordenadores y data show en todas las aulas. Asimismo, en su mayoría reconocen que ha habido una permanente preocupación por renovar equipos y por mejorar la velocidad de conexión a Internet, pues en el último tiempo tienen la posibilidad de conectarse a la Red en gran parte de los espacios físicos.

En relación con la infraestructura y recursos disponibles, también destacan que no tienen dificultades para acceder al uso de los ordenadores, salvo en determinados horarios en que el Laboratorio de Computación está ocupado con clases. Del mismo modo valoran el hecho de que pueden acceder al préstamo de equipos portátiles, donde principalmente se ven favorecidos los estudiantes que se encuentran realizando su trabajo final de titulación. También se aprecia, sin embargo, que desde su perspectiva no ocurre lo mismo al solicitar el préstamo de otro tipo de recursos tecnológicos o herramientas audiovisuales, pues manifiestan que no es fácil poder acceder a ellos.

En cuanto a la forma en cómo perciben las prácticas de los docentes con TIC, los estudiantes enfatizan que la mayoría del profesorado solamente utiliza clases tradicionales, donde la presentación de contenidos por medio del Power Point sería la única aproximación al uso de las tecnologías, inclusive subrayan que existe un abuso de esta herramienta. Para los estudiantes la razón de esta práctica se fundamenta en que los docentes no tendrían conocimientos sobre todas las posibilidades educativas que ofrecen actualmente las TIC y cómo poder aplicarlas didácticamente en el aula, lo que les ha repercutido en su propia 
formación y, específicamente, en la adquisición de conocimientos sobre el uso educativo de estas herramientas.

Por otra parte, valoran positivamente la asignatura llamada Tecnologías y Aprendizaje, que curricularmente está programada para el primer semestre del periodo formativo. No obstante, enfatizan en que al ser la única asignatura vinculada a las TIC, y, además, el hecho de estar ubicada al inicio de su carrera y no tener continuidad con el transcurso del tiempo, hace que se les haya ido olvidando lo que allí aprendieron, aspecto que han comenzado a valorar de manera significativa cuando se han visto enfrentados a su práctica profesional y les ha surgido la necesidad de hacer uso de estas herramientas en sus clases. Por lo anterior, sugieren que se analice la continuidad de esta asignatura en la malla curricular o que se incorpore otra asignatura donde se aborde el desarrollo de competencias que les permitan hacer uso de estas herramientas en forma didáctica, pero en el periodo previo a la práctica profesional (ver Tabla 9).

Tabla 9. Evidencias subcategorías factores contextuales

\begin{tabular}{|c|c|}
\hline Subcategorías & Extractos textuales \\
\hline $\begin{array}{l}\text { Implementación de } \\
\text { TIC en la Facultad }\end{array}$ & $\begin{array}{l}\text { "yo siento que la Universidad está un poco estancada en cuanto a } \\
\text { tecnología, como que llegaron a un punto y dijeron iy hasta aquí } \\
\text { llegamos!, pero la tecnología cada vez va avanzando más, entonces la } \\
\text { misma pizarra digital que dice mi compañero son tecnologías que se están } \\
\text { implementando hace poco y siento que la universidad se quedó estancada. } \\
\text { Tenemos Wifi y ya!!" (PC6). } \\
\text { "Yo encuentro que en ese sentido la Universidad abarca varias de las } \\
\text { necesidades, en el sentido de que hay Internet, hay computadores, a las } \\
\text { personas que no tienen notebook les prestan, entonces en ese sentido han } \\
\text { pensado mucho en las necesidades de los alumnos, y para ser una } \\
\text { Universidad pequeña que tal vez no tenga tanto financiamiento como } \\
\text { otras, cumple con las demandas que tienen los alumnos" (PCN4). }\end{array}$ \\
\hline $\begin{array}{l}\text { Accesibilidad a los } \\
\text { recursos disponibles }\end{array}$ & $\begin{array}{l}\text { "[...] a veces uno va a pedir un computador y no se puede o que están con } \\
\text { problemas, o que si quieres buscar algún tipo de documento el Internet no } \\
\text { va con la velocidad que debería ir" (PEGB3). } \\
\text { "[...] el año pasado recién cambiaron los equipos del Laboratorio, yo creo } \\
\text { que tenemos acceso a eso y tenemos el Wifi y todo, entonces yo encuentro } \\
\text { que tenemos la herramienta como para trabajar" (PI5). } \\
\text { "[...] yo encuentro, en términos personales, que acceder a los } \\
\text { computadores u otros recursos no me parece tan complejo ni tan } \\
\text { engorroso, dependiendo del horario, pero lo que siempre es un lío es } \\
\text { imprimir" (PEGB2). } \\
\text { "[...] yo creo que nadie tiene que quejarse ni decir nada porque es súper } \\
\text { fácil pedir un computador, la sala de computación siempre está disponible, } \\
\text { no nos ponen obstáculos" (PEP2). }\end{array}$ \\
\hline
\end{tabular}




\begin{tabular}{|c|c|}
\hline $\begin{array}{l}\text { Prácticas docentes } \\
\text { con TIC }\end{array}$ & $\begin{array}{l}\text { "En Didáctica del Cálculo la mayoría de las presentaciones las hacían con } \\
\text { Geo y el profesor nos habló del Geo en una clase, nos hizo una pequeña } \\
\text { presentación del programa y nos dijo trabajen con este programa que es } \\
\text { bueno, y eso fue todo. La mayoría trabajó con el programa, pero no fue } \\
\text { una clase para utilizar el programa" (PEM1). } \\
\text { "[...] yo por lo menos nunca he visto una pizarra digital, no la enseñaron } \\
\text { a utilizar ni nada. Sé que la Universidad cuenta con una, pero nadie te } \\
\text { enseña a trabajar con ella" (PC2). } \\
\text { "[...] acá, por ejemplo, los profesores son } 50 \text { diapositivas en clases, } 40 \text { e } \\
\text { incluso } 60 \text { y puro texto, entonces yo creo que va en la tercera diapositiva } \\
\text { y ya lo único que uno quiere es irse de aburrido" (PCN1). } \\
\text { "Yo creo que cuando hablamos de las tecnologías todos pensamos de } \\
\text { inmediato en un Power Point, acá por ejemplo no se aplica otro método o } \\
\text { es la clase tradicional o con el Power Point" (PCN3). } \\
\text { "La mayoría de las asignaturas son clases tradicionales, no se ocupan } \\
\text { mucho las tecnologías, aparte de las presentaciones Power Point" (PCN2). }\end{array}$ \\
\hline $\begin{array}{l}\text { Aspectos } \\
\text { curriculares }\end{array}$ & $\begin{array}{l}\text { "Por qué hacer un ramo que te sirve tanto [alusión a la asignatura de } \\
\text { Tecnología y Aprendizaje], tenerlo en primer año cuando tú tienes tan } \\
\text { poco conocimiento de la matemática que te van a pasar más adelante?" } \\
\text { (PEM1). } \\
\text { "Yo creo que si no estuviera ese ramo en primer año realmente no lo } \\
\text { echaría de menos [haciendo alusión a la asignatura de Tecnología y } \\
\text { Aprendizaje] Yo siento que no aprendimos nada en ese ramo, que debería } \\
\text { haber estado más adelante antes de que fuésemos a empezar la práctica } \\
\text { pedagógica para habernos preparado mejor y con otras herramientas para } \\
\text { trabajar con los estudiantes, no solo con Excel y con Power Point" (PC2). } \\
\text { "Se aprende mucho en Tecnología y Aprendizaje, pero lo único malo es } \\
\text { que es en primero, entonces uno ahora ya no se acuerda mucho de lo que } \\
\text { aprendió" (PCN4). } \\
\text { "Yo pienso que ese ramo debería estar al inicio de la carrera y al final, } \\
\text { [haciendo alusión a la asignatura de Tecnología y Aprendizaje] cuando se } \\
\text { está por ir a la práctica, porque al inicio nos enseñaron a utilizar el Excel } \\
\text { y eso yo encuentro que fue súper útil para la carrera porque en casi todos } \\
\text { los ramos nos pedían gráfico, pero también debería estar casi al final, } \\
\text { antes de la práctica donde nos enseñaran a utilizar estas herramientas } \\
\text { dirigidas a lo que es el aprendizaje y a la enseñanza" (PCN2). }\end{array}$ \\
\hline
\end{tabular}

Nota: PC: Pedagogía en Castellano y Comunicación; PEP: Pedagogía en Educación Parvularia; PM: Pedagogía en Educación Matemática; PEGB: Pedagogía en Educación General Básica; PCN: Pedagogía en Ciencias Naturales. Cada número que acompaña a la sigla representa al estudiante al cual se le asignó dicho número.

\section{DISCUSIÓN Y CONCLUSIONES}

Dentro de las competencias que una persona necesita desarrollar hoy en día se encuentra la competencia digital, entendida esta como la movilización de aquellas habilidades y destrezas que permiten buscar, seleccionar críticamente, obtener y procesar información relevante haciendo uso de las TIC para transformarla en conocimiento. Al mismo tiempo, debe ser capaz de comunicar dicha información a través de la utilización de diferentes 
soportes tecnológicos y digitales, actuando con responsabilidad, respetando las normas socialmente establecidas y aprovechando estas herramientas para informarse, aprender, resolver problemas y comunicarse en distintos escenarios de interacción. Por tanto, el sentido dado a la competencia digital en este estudio va más allá de poseer únicamente la capacidad para utilizar las herramientas tecnológicas disponibles.

En efecto, actualmente no basta con que los futuros maestros logren adquirir las competencias sobre el manejo de estas herramientas, pues, fundamentalmente se requiere que sean capaces de articular dichos conocimientos con los saberes didácticos específicos de su ámbito disciplinario.

En respuesta a ambos propósitos, se puede decir que para los estudiantes las TIC han adquirido gran relevancia en su vida personal y académica, pues reconocen la necesidad de hacer uso de ellas para estar comunicados, acceder a la información que requieren y cumplir con las exigencias demandadas por los docentes. Asimismo, demuestran una disposición muy favorable para que estas herramientas sean incorporadas a la educación, ya que para ellos facilitan la labor docente en todas sus dimensiones, resultado que está en la línea de otros estudios (Arras et al., 2011; Puentes et al., 2013).

El significado que han ido construyendo respecto a las TIC se basa fundamentalmente en la experiencia que han obtenido a lo largo de su proceso formativo, donde escasamente han tenido oportunidad de utilizar otro tipo de dispositivos o recursos digitales que actualmente están disponibles, por ejemplo, al acceder a la Web 2.0. Ello es debido a que los docentes responsables de la formación se han enmarcado dentro de modelos educativos tradicionales, donde las TIC utilizadas como recurso didáctico han sido escasamente valoradas. En gran medida este aspecto se ha constituido en una de las cuestiones clave que no solo ha determinado el significado atribuido por los estudiantes a estas herramientas, sino que a la vez se ha transformado en uno de los factores que ha generado la adquisición de conocimientos básicos e instrumentales sobre su utilización y un escaso conocimiento sobre cómo aplicar las herramientas tecnológicas y digitales desde una perspectiva pedagógica tal y como se apuntaba en el estudio realizado por Ribot, Llach y Cicres (2010).

Otro aspecto que está vinculado al bajo nivel de conocimientos en el uso de TIC como recurso didáctico autopercibido por los estudiantes corresponde a su Plan de Estudios, ya que este solo comprende una asignatura vinculada a estas herramientas en el primer año de formación, cuya duración es de un semestre y se descontinúa por completo a lo largo del resto del proceso. Además, se focaliza en alfabetizar tecnológicamente a los estudiantes en aquellas cuestiones básicas que necesitarán durante su proceso formativo: por ejemplo, el uso básico de Word, Power Point y Excel. Esta característica curricular genera ciertas limitaciones para ofrecer a los estudiantes otras instancias donde puedan aprender a hacer uso de las herramientas tecnológicas y digitales disponibles, y aprovecharlas en un sentido educativo. Para Duhaney (2001 Cit. en Silva y Astudillo, 2012, p. 4), esta situación curricular se transforma en una barrera para que los futuros maestros integren estas herramientas en el aula. Por otra parte, se puede sostener que a los estudiantes no solo les ha repercutido la no continuidad de una asignatura en particular, sino que, a la vez, también ha sido determinante el hecho de que las TIC no sean incorporadas de manera transversal en el resto de las asignaturas, resultado coincidente con otros estudios (Gallego et al., 2010; Vaillant, 2014).

Finalmente, no se puede dejar de mencionar que el factor personal de los estudiantes también se ha constituido en un aspecto clave sobre el nivel de competencias en TIC que 
han logrado adquirir, fundamentalmente la actitud positiva que demuestran frente a estas herramientas, además del interés por aprender a utilizarlas, pues manifiestan que han aprendido a usarlas mayoritariamente en forma autodidacta, resultado que es compatible con el de otros estudios (Centeno y Cubo, 2013; Cózar y Roblizo, 2014). No obstante, se puede sostener que la postura de ellos es más bien pasiva respecto a querer aprender a utilizarlas con un mayor nivel de conocimientos o bien otorgarles una orientación didáctica. Esta idea que se fundamenta en el hecho de que dadas las posibilidades que ofrece hoy en día la Web 2.0 para acceder a recursos educativos digitales y multimedia variados, para acceder a múltiples aplicaciones y software que permiten crear recursos educativos propios — como, por ejemplo, blogs, sitios web, recursos multimedia, etc.— los estudiantes solo se remiten al uso de procesadores de texto, planillas de cálculo y presentaciones tradicionales.

Asimismo, se destaca que lo aprendido al inicio de su proceso formativo en la asignatura específica sobre TIC — Tecnologías y Aprendizaje - solo respondió a una práctica llevada a cabo para cumplir con las exigencias de aprobación de la misma, pues no continuaron actualizando, practicando o aplicando esos aprendizajes al resto de las asignaturas, razón que los ha llevado a no transferir lo que allí aprendieron. Esta situación induce a sostener que los estudiantes presentan cierto grado de incapacidad para reflexionar sobre su responsabilidad en el olvido de dichos aprendizajes, debido a que para ellos los docentes serían los principales responsables de las carencias que presentan respecto a la aplicación didáctica de las TIC.

Hoy en día se está evidenciando un mayor interés sobre lo que está pasando en la formación inicial del profesorado respecto a la implementación de estrategias que ayuden a la optimización del proceso formativo de los futuros maestros como una forma de avanzar hacia la mejora de la educación en los distintos niveles del sistema educativo. Por otra parte, las TIC se están valorando como herramientas que pueden constituirse en recursos que ayuden a la generación de ambientes de aprendizaje enriquecidos y contextualizados a la realidad que los niños y jóvenes están viviendo. Al conjugar estos dos aspectos se puede desprender que las demandas sociales actuales exigen a las instituciones de Educación Superior que los futuros maestros egresen siendo educadores competentes no exclusivamente en el dominio de los conocimientos pedagógicos y disciplinarios, sino que, además, sean competentes en el dominio de conocimientos tecnológicos aplicados a la educación.

\section{REFERENCIAS BIBLIOGRÁFICAS}

Adell, J. (2010). Dimensiones de la competencia digital. La Competencia Digital. XXIV Jornadas Pedagógicas de Barakaldo. Recuperado de https:/www.youtube.com/watch?v=qWLTMr6ZmzM

Arras, A.M., Torres, C., \& García-Valcárcel, A.M. (2011). Competencias en Tecnologías de Información y Comunicación (TIC) de los estudiantes universitarios. Revista Latina de Comunicación Social, 66, 130-152.

Badilla, M. G., Jiménez; L., \& Careaga, M. (2013). Competencias TIC en formación inicial docente. Aloma. Revista de Psicología, Ciències de l'Educació i de l'Esport, 31(1), 89-97.

Bisquerra, R. (Coord.). (2014). Metodología de la Investigación Educativa. Madrid: La Muralla.

Brun, M. (2011). Las tecnologías de la información y comunicaciones en la Formación Inicial Docente en América Latina. CEPAL. Serie Políticas Sociales, 172, Santiago de Chile. Recuperado de http://hdl.handle.net/11362/6183 
Cabero, J., \& Marín, V. (2014). Miradas sobre la formación del profesorado en tecnologías de información y comunicación (TIC). Enl@ ce: Revista Venezolana de Información de Tecnología y Conocimiento, 11(2), 11-24.

Centeno, G., \& Cubo, S. (2013). Evaluación de la competencia digital y las actitudes hacia las TIC del alumnado universitario. Revista de Investigación Educativa, 31(2), 517-536. doi:10.6018/ rie.31.2.169271

Cózar, R., \& Roblizo, M. (2014). La competencia digital en la formación de los futuros maestros: percepciones de los alumnos de los Grados de Maestro de la Facultad de Educación de Albacete. Revista Latinoamericana de Tecnología Educativa, 13(2), 129-133.

De Saint Pierre, D. (2008). Futuros profesores: ¿por qué formar en competencias digitales? Chile: Enlaces. Ministerio de Educación. Recuperado de http://enlacesenred.blogspot.com.es/2008/10/ futuros-profesores-por-qu-formar-en.html

Fainholc, B., Navia, H., Romero, R., \& Halal, C. (2013). La formación del profesorado y el uso pedagógico de las TIC. Revista de Educación a Distancia, 38, 1-14. Recuperado de http://www. um.es/ead/red/38/fainholc.pdf

Flores, C. (2014). Competencia digital docente: desempeños didácticos en la formación inicial del profesorado. Revista científica de educación y comunicación. Hachetetepe, 9, 55-70. Recuperado de http://revistaeducarnos.com/wp-content/uploads/2014/11/Libro-Nov.14-web.pdf

Gallego, M.J., Gámiz, V., \& Gutiérrez, E. (2010). El futuro docente ante las competencias en el uso de las tecnologías de la información y comunicación para enseñar. EDUTEC, Revista de Tecnología Educativa, 34. Recuperado de http://edutec.rediris.es/Revelec2/revelec34/futuro_ docente_competencias_tic.html

Gisbert, M., Espuny, C., \& González, J. (2011). INCOTIC. Una herramienta para la @ utoevaluación diagnóstica de la competencia digital en la universidad. Profesorado: revista de currículum y formación de profesorado, 15(1), 76-89.

Gutiérrez, A., Palacios, A., \& Torrego, L. (2010). La formación de los futuros maestros y la integración de las TIC en la educación: anatomía de un desencuentro. Revista de Educación, 352. Recuperado de http://www.revistaeducacion.educacion.es/re352/re352_TIC.pdf.

Gutiérrez, I. (2014). Perfil del profesor universitario español en torno a las competencias en tecnologías de la información y de la comunicación. Pixel-Bit. Revista de Medios y Educación, 44, 51-65. doi:10.12795/pixelbit.2014.i44.04

Llorente, $\mathrm{M}^{\mathrm{a}}$ del C., Barroso, J., \& Cabero, J. (2015). Las tecnologías de la información y la comunicación: principios para su aplicación, integración y selección educativa. En J. Cabero y J. Barroso (Coords.), Nuevos retos en tecnología educativa (pp. 41-67). Madrid: Editorial Síntesis.

Martínez, F. (2009). Mitología de las TIC en la sociedad y en la enseñanza. Educatio Siglo XXI, 27(2), 33-42. Recuperado de http://revistas.um.es/educatio/article/view/90941/87721

Pino, M., \& Soto, J. (2010). Identificación del dominio de competencias digitales en el alumnado de grado de magisterio. Teoría de la Educación. Educación y Cultura en la Sociedad de la Información (TESI), 11(3), 336-362. Recuperado de http://campus.usal.es/ revistas_trabajo/ index.php/revistatesi/article/view/7466/7483

Prendes, M.P., Castañeda, L., \& Gutiérrez, I. (2010). Competencias para el uso de TIC de los futuros maestros. Comunicar, Revista Científica Iberoamericana de Comunicación y Educación, XVIII(35), 175-182. doi:10.3916/C35-2010-03-11

Puentes, A., Roig, R., Sanhueza, S., \& Friz, M. (2013). Concepciones sobre las Tecnologías de la Información y la Comunicación (TIC) y sus implicaciones educativas: Un estudio exploratorio con profesorado de la provincia de Nuble, Chile. Revista Iberoamericana de Ciencia, Tecnología y Sociedad, 8(22), 75-88. Recuperado de http://rua.ua.es/dspace/bitstream/10045/35183/1/2013_ Puentes_etal_CTS.pdf

Quintanal, J., \& García, B. (Coords.). (2012). Fundamentos básicos de metodología de investigación educativa. Madrid: Editorial CCS. 
Ribot, M. D., Llach, S., \& Cicres, J. (2010). La incidencia de las nuevas tecnologías en los hábitos formativos de los estudiantes de Magisterio. Pixel-Bit. Revista de Medios y Educación, 37, 107120. Recuperado de http://www.sav.us.es/pixelbit/pixelbit/articulos/n37/9.pdf

Roig, R., \& Pascual, A.M. (2012). Las competencias digitales de los futuros docentes. Un análisis con estudiantes de Magisterio de Educación Infantil de la Universidad de Alicante. @ tic. revista d'innovació educativa, 9, 53-60. doi:10.7203/attic.9.1958

Silva, J., \& Astudillo, V. (2012). Inserción de TIC en la formación inicial docente: barreras y oportunidades. Revista Iberoamericana de Educación, 58(4), 1-11. Recuperado de http://www. rieoei.org/deloslectores/4557Silva.pdf

Vaillant, D. (2013). Integración de TIC en los sistemas de formación docente inicial y continua para la Educación Básica en América Latina. Argentina: Fondo de las Naciones Unidas para la Infancia (UNICEF). Recuperado de http://www.unicef.org/argentina/spanish/educacion_ Integracion_TIC_sistemas_formacion_docente.pdf

Vaillant, D. (2014). Formación de profesores en escenarios TIC. Revista e-Currículum, 2(12), 1128 1142. Recuperado de http://goo.gl/lHYB2y

Valverde, J., Garrido, M.C., \& Fernández, R. (2010). Enseñar y aprender con tecnologías: Un modelo teórico para las Buenas Prácticas con TIC. TESI, 11(3), 203-229.

Vílchez, J., \& Pérez, F. (2013). Percepción de Futuros Maestros sobre el potencial de las Tic en la Educación: de las Expectativas a la Realidad. Revista Fuentes, 13, 155-172. Recuperado de http://institucional.us.es/fuentes/gestor/apartados_revista/pdf/campo/fazwddpk.pdf 